\title{
CON-KER: A CONVOLUTIONAL NEURAL NETWORK BASED APPROACH FOR KERATOCONUS DETECTION AND CLASSIFICATION
}

\author{
Shashank $\mathrm{V}^{1}$, Priya $\mathrm{D}^{2}$,Dr. G S Mamatha ${ }^{3}$, Dr Nagaraju $\mathrm{G}^{4}$ \\ ${ }^{1}$ Post Graduate Student, Information Technology, R V College of Engineering, Bengaluru - 560059. \\ ${ }^{2}$ Assistant Professor, Department of Information science and Engineering, $R V$ College of Engineering, \\ Bengaluru - 560059. \\ ${ }^{3}$ Professor\& Dean (PG studies), Department of Information science and Engineering, $R$ V College of \\ Engineering, Bengaluru - 560059. \\ ${ }^{4}$ Professor, Minto ophthalmic hospital, Bengaluru -560004
}

${ }^{1}$ Email: shashankv.sit19@rvce.edu.in

\begin{abstract}
The paper is on detection of keratoconus a corneal progressive disorder leading to the thinning and also protrusion of the cornea associated with symptoms like astigmatism, increased sensitivity to bright light, glare, clouded vision, eye irritation and others, recent times there has been increase in number of keratoconus cases. Keratoconus is normally described as a non-inflammatory pathology. The main contribution of the paper is to facilitate detection and also classification of the keratoconus based on the progression using Convolution neural networks. The paper is about the implementation of different CNN algorithms which will classify the disorder based on the progression into 4 different classes. The CNN algorithms analyze corneal topography if the eye and classify based on the severity of the disorder. We introduce an effective CNN model called CON-KER for the detection and classification of the disorder. Further CNN algorithms like Alexnet and Vgg 19 were implemented for the same. The results show that CON-KER model has yielded the accuracy of $96.26 \%$ comparedto other algorithms like vgg19 which yielded $94.76 \%$ and AlexNet with $86 \%$ accuracy. This work can help by assisting the ophthalmologist in reducing he diagnostic errors and also help in rapid screening of the patients.
\end{abstract}

Index Terms - Convolutional neural network (CNN), Keratoconus 19, Alex Net, Vgg, Topography,CONKER

\section{Introduction}

Keratoconus is associated with the corneal part of the eye; cornea is the outer covering layer of the front eye. Cornea does not contain blood vessels it is made of proteins and cells. membrane protecting the inner part of eye and also help in focusing the light so that a person can have a clear vision. There are several conditions which is observed commonly affecting the cornea including scratches which heal overtime on their own but cornea which is highly scarred is also seen in the patients of keratoconus. Cornea does not contain blood vessels it is made of cells and proteins. The thinning which is progressive and therefore the resulting bulging of the corneal part of the eye are typically in the midst of high myopic, accompanied by irregular astigmatism, leading to severe visual impairment. Keratoconus affects throughout the second decade of one's life sometimes once a patient is socially active[1,2,21].

Reduced vision leads many patients to have refractive surgery due to severity of their symptoms via LASIK surgery $[4,6,16,34,35,24]$. Corneal topographic sans are used in screening. Topography presentscolor maps in "absolute" and "normalized" scales. The topography offersa top-level view of the complete cornea in phrases of its curvature. The carried outset of rulesmethodsregular corneal topographies and classifies them into categories, detecting stylesunique to the keratoconus pathology [5]. In recent years early diagnosis has become extremely relevant because diagnosis is mandatory to avoid surgery [7]. Development of more sensitive algorithms for the detection of most incipient cases is of current of great interest as there are several therapeutic options that would allow giving a check to the progression of the disorder [8]. The work introduces a CNN model named CON-KER 
which aid in detection and classification of Keratoconus. Other well-known algorithms like Alexnet and vgg19 were used for the detection and classification of the disorder.

In the course of recent years, convolution neural networks have made a major impact on detection and classification of different eye diseases and also other diseases like cancer tumors [22,37]. The CNN algorithms effectively detect and classify images.CNN algorithms have a great potential in for use in the medical field [23], it reduces screening time and also increases the accuracy of the detection of the disease.

Causes of the keratoconus is predicted to be many, it can be genetically acquired, genetic factors are linked to ethnicity as well in countries like India, Iran have high proportion of population effected by keratoconus $[12,18,28,29]$ in $10-28 \%$ patient have family history of keratoconus and multiple genes are involved in the development of the disorder [15,31-33]. Keratoconus can cause due to age, there are also certain disorders which might have connection to causing keratoconus such as down's syndrome, osteogenesis and few others, inflammation from asthma, allergies and atopic diseases of eye said be known to breakdown the tissues of the cornea. Eye rubbing over longer period of time can break the cornea and corneal scarring is usually observed in keratoconus patients. One in 2000 person is affected by keratoconus but this is increasing significantly from the recent past. Patients are seen to develop double vision, blurry vision, light streaks, glare, eye irritation and few other symptoms. Patients can be treated at the right time avoiding further complications and also result in detecting and providing early proper cure for the disease. Keratoconus needs different treatments based on the progression of the disorder, mild cases are usually corrected with eyeglasses and contact lenses, corneal crosslinking with UV light and riboflavin, cases of keratoconus which are advance require surgical treatment which includes corneal transplant of the cornea from a donor, but there is risk of failure where the eye rejects the donor's cornea. To avoid all these complications, early detection and correction of the disorder is recommended $[17,36]$, the work aids in early detection and classification of keratoconus based on the corneal topographic maps. Detection of subclinical and mild keratoconus is a priority to control the disorder as the progression of the disease clearly lead to treatment like corneal transplant which is unsuccessful in many cases as the eye rejects the cornea, the disorder when detected at the earliest stage is one of the main objectives of the work, the inclusion of the mild keratoconus scans and done ant the models are trained so that they can detect and classify mild keratoconus from normal eye.

\section{Related Work}

Keratoconus is one such disorder on which many studies are being done in recent years, as the number of cases is increasing the studies and methods of screening based on machine learning is increasing. The previous works is majorly done using raw data which contains readings based on corneal scans, Inclusion of corneal topographic scans for the detection and classification of keratoconus. Detection of mild and subclinical keratoconus is a challenging task.

A very few studies are done based on CNN based classification of the disorder.

(1) A CNN based algorithm was proposed in [11] by analyzing the raw data of the Pentacam HR system for detection of the subclinical keratoconus by considering an inhouse dataset, here attempt is made to collect a raw data of specific format with 5 numerical matrices, the work mainly concentrates on detection of Subclinical keratoconus. The work considers 854 samples including both men and women of age around 20-30 years of age. The work proposes end to end deep learning approach.

(2) Lavric, Valetin [5] proposes a CNN based algorithm for the detection of keratoconus, the work considers 3000 topographic scans, the Pentacam based scans were used to classify the disorder as normal eye and keratoconus, this work was first of its kind Implementing CNN methodology into detection of the disorder.

(3) Toutounchian[3] implemented artificial intelligence algorithm for the detection of the disorder using the topographic maps ,Considering 82 topographic scans, 12 features from each map were considered and different algorithms like neural network, SVM, Multilayer perceptron and decision tree.

(4) Authors of [41] use a customized model for detection Keratoconus by using raw data and few selected parameters, a feed forward network is used to apply on Pentacam based data, the work doesn't use end to end method in the detection of the disorder

(5) Apart from Pentacam based data used for detection of the disorder Siamak yousefi[1] uses SS-1000 CASIA OCT images , 12,242 images which was further divided based on pachymetry , elevation , Ectasia static index and propose and algorithm towhich uses principal component analysis, manifold learning and density based clustering to identify the severity of keratoconus. 


\section{DATASET}

Keratoconus can affect all layers of the cornea. The epithelial cells of the cornea may be elongated and enlarged $[14,30]$.Keratoconus is diagnosed by considering topographic scans of the cornea, we consider the topographic maps of the cornea in this work as the data, corneal topography helps in mapping the anterior surface of the cornea and it is also a non-invasive technique, a computer assisted tool creates a map of the surface if the cornea which further can help to analyse different corneal parameters.

Corneal topographic maps are clinical data which is difficult to obtain so with the minimum available images the data augmentation technique is used to generate the corneal topographies. Keratoconus is a disorder which may affect single eye or both eyes. Patterns which usually occur in the case of keratoconus are revealed by corneal topographic maps. Validation of the dataset for further classification with the help of an ophthalmologist for the classification of the dataset into different stages of keratoconus and also understanding different profiles used in the scans was done. The dataset majorly consisted of 2 different profiles which were curvature profile and thickness profile of the cornea, the dataset was divided into 4 different classes like normal eye, Mild keratoconus, Moderate keratoconus and advanced keratoconus.

Topographic maps use a colour scale to identify corneal curvature data. Curved steep regions are displayed in heat colours, that embrace pink and orange, whereas flat bend regions are evidenced in bloodless colours, which involve blue and green [9]. Keratoconus can affect the population of different ages it may start at an early age and progress and worsen the vision, but the progression must be stopped with early diagnosis and treatment. These 4 classes were considered based on the thickness and the curvature profiles of the cornea.The thickness range (measured in $\mu \mathrm{m}$ ) and the curvature are measured in Dioptres (D).

In table 1 and 2, the ranges of the thickness and the curvature based on the 4 class is presented.

Table 1 Corneal thickness profile range

\begin{tabular}{|l|l|}
\hline \multicolumn{1}{|c|}{ Thickness range (pachymetry) } & \multicolumn{1}{c|}{ In $\mu \mathrm{m}$} \\
\hline Normal eye & $>520$ \\
\hline Mild Keratoconus & $500-520$ \\
\hline Moderate Keratoconus & $300-500$ \\
\hline Advanced Keratoconus & $<300$ \\
\hline
\end{tabular}

Table 2 Corneal curvature Profile

\begin{tabular}{|l|l|}
\hline Curvature & In Diopters \\
\hline Normal eye & $<43 \mathrm{D}$ \\
\hline Mild Keratoconus & $43-48 \mathrm{D}$ \\
\hline Moderate Keratoconus & $48-53 \mathrm{D}$ \\
\hline Advanced Keratoconus & $>53 \mathrm{D}$ \\
\hline
\end{tabular}




\section{METHODOLOGY}

The main aim of the work is to implement Convolution neural network algorithms in detection and classification of the corneal disorder keratoconus. In the implementation the goal is also to detect the mild keratoconus which will lead to early correction and proper treatment, the neural networks used take the topographic data which is labelled into 4 classes based on the severity of the disease which is normal eye, mild, moderate and advanced keratoconus. The images considered were in the jpg format and all images were the topographic scans of the cornea. We implement and introduce CON-KER CNN model and also two others well know models like VGG 19 and Alex net for the comparison study.

Many learning algorithms use multilayer networks between inputs and outputs. These neural networks allow the identification of features, patterns, and characteristics within the classification relationship. Technological progress has led to the development and refinement of these algorithms, with them being used in many areas of medicine with confidencein neural networks, a convolutional neural network (CNN) is one of the main methods of recognizing and classifying images. CNNs are currently used in applications such as object recognition and face detection. A CNN that is capable of diagnosing the keratoconus disease is implemented in this paper [5]. The idea of the work is represented in fig 1

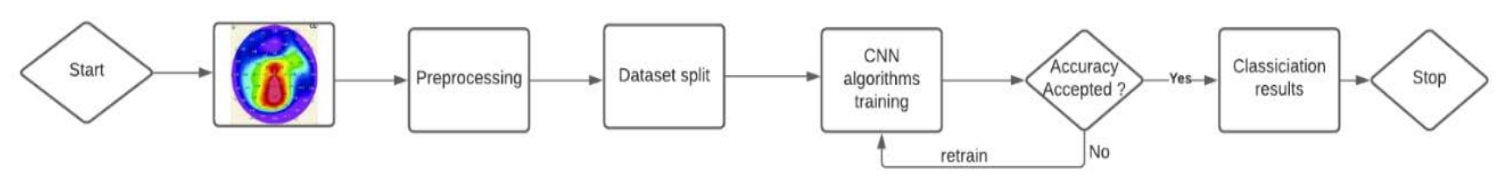

Fig 1 Proposed workflow

Before introducing the image into the convolution neural network, it undergoes pre-processing step, the input images should be made sure that they are of the same size and format, the image which topographic maps considered as the dataset for the classification.

\section{CON-KER}

CON-KER is a new model introduced for the detection and classification of the corneal disorder. The introduced CNN model effectively detects and classifies corneal topographic maps into different stages of Keratoconus.

CON-KER is effective CNN model which uses color images of size $180 \times 180 \times 3$. The CON-KER neural network has different layers convolutional blocks with maxpooling layer associated to each, the other layers are flatten layer and fully connected layers and output layer. The CNN structure is implemented using python code. This is a sequential model and the layer is shown in fig 2

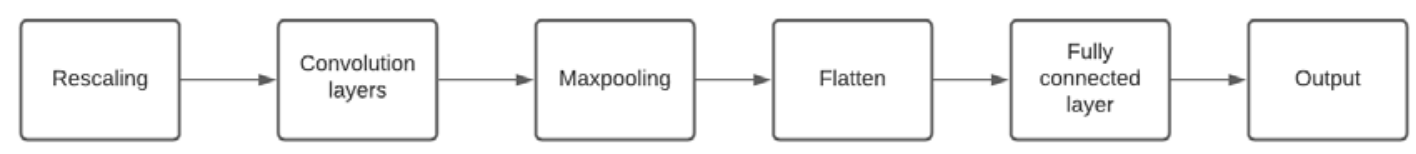

Fig 2 Brief architecture of CON-KER

2071 Topographic maps were obtained with augmentation technique in which 1657 files used for training divided into 4 classes, 414 for validation. The algorithm is trained to classify the keratoconus into 4 classes including the normal eye. The neural network yielded an accuracy of $96.26 \%$ which is further explained in the result section.

\section{Alexnet}

AlexNet is a deep convolutional neural networkconsists of convolutionallayers, sub samplinglayers and fullyconnected layers. By using AlexNet we can train more than a million images from thedatabase [10]. AlexNet, it was the second algorithm used for the keratoconus detection and classification. AlexNet is a 
pertained and an efficient model which has been used for detection and classification of several eye diseases in the past. AlexNet is the convolution network with 5 convolution layers ,3 max pooling layers and 2 fully connected layers, the AlexNet is a pre trained model which has 650000 neurons with 60million parameters it can handle 1000 classes but there is a fear of overfitting so data augmentation is the easiest way to solve the issue of overfitting. Here the AlexNet is used for detection and classification of Keratoconus. The AlexNet model was trained with the topographic maps, the trained model yielded and accuracy of $88.90 \%$ and validation accuracy of $84.78 \%$.

\section{VGG}

vgg is a classical convolutional neural network architecture. It is primarily based totally on evaluation of the way to growth the intensity of such networks. The network makes use of small three $x$ three filters. Otherwise, the community is characterized with the aid of using its simplicity: the most effective different additives being pooling layers and a completely linked layer [11]. Vgg 19 is a CNN model that consists of 16 convolution layers, 5 Max pool, 3 fully connected and 1 SoftMax layer.Vgg net is the successor of the AlexNet .Vgg19 takes a fixed size image (RGB) input of size 224 x 224 x 3 pixels. Similar to AlexNet VGG is also used for detection and classification of medical images. Vgg 19 was trained with the topographic maps of curvature and thickness profiles similar to the other models, the neural network post training gave and accuracy of $94.76 \%$ and a validation accuracy of $77.05 \%$.

\section{RESULTS}

This section describes the results obtained and the plots related to the obtained results. The CNN models were trained with the topographic maps and accuracy and loss were derived, each model has a different accuracy and validation scores. Fig 3 shows the CON-KER sequential model training vs validation accuracy plot, the validation accuracy with respect to the epochs, each of the model were trained and plots were obtained. The fig 4 represents the accuracy score for training vs validation, for 15 epochs each.The CON-KER Convolution neural network accuracy (validation vs training), the CON-KER neural network yielded a training accuracy of the $96.2 \%$ vs validation accuracy of $94.6 \%$ the loss of 0.24 loss for validation and 0.09 loss for training value for 15 epochs.

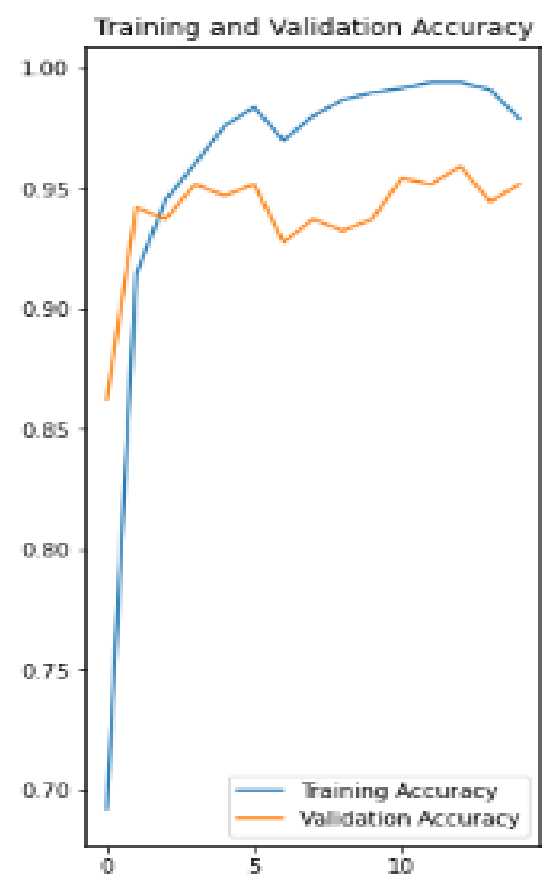

Fig 3 CON-KER sequential model training vs validation accuracy plot 
The fig 3 shows the training vs validation loss for the CON-KER sequential model of the CNN proposed for detection and classification of keratoconus using CNN.

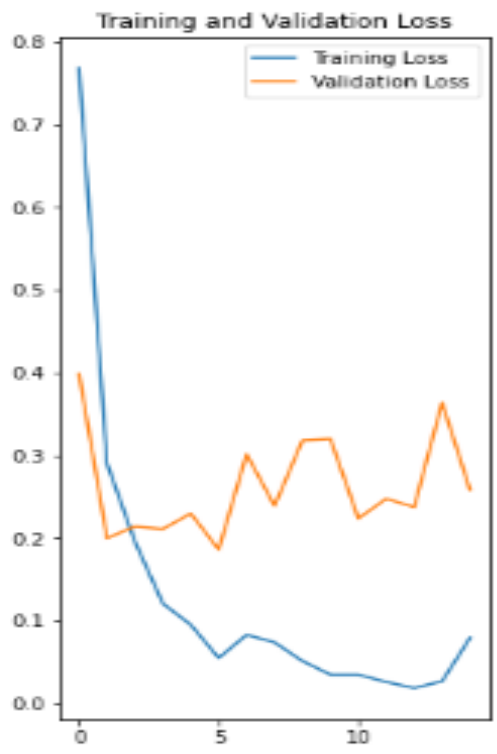

Fig 4 Training vs validation loss plot for CON-KER model

Alex net accuracy scores are described in this section, Alex Net was the next model used for the detection and classification of Keratoconus, the model was trained and the training accuracy and loss: 0.7665 - accuracy: 88.90\% - validation loss: 0.5551 - Val accuracy: $84.78 \%$. The fig 5 and 6 represents the plot of validation vs training accuracy and validation vs training loss.

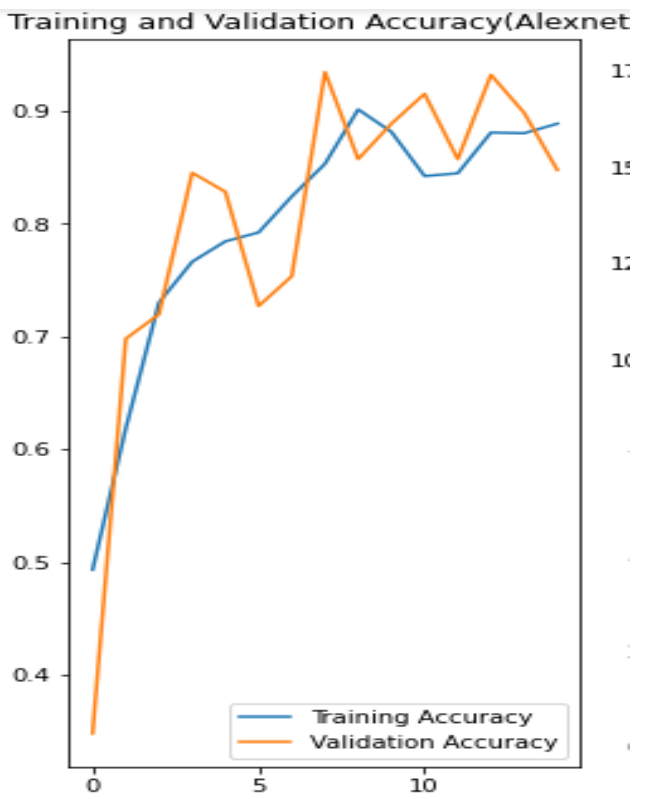

Fig 5 Training vs Validation accuracy plot of Alexnet 


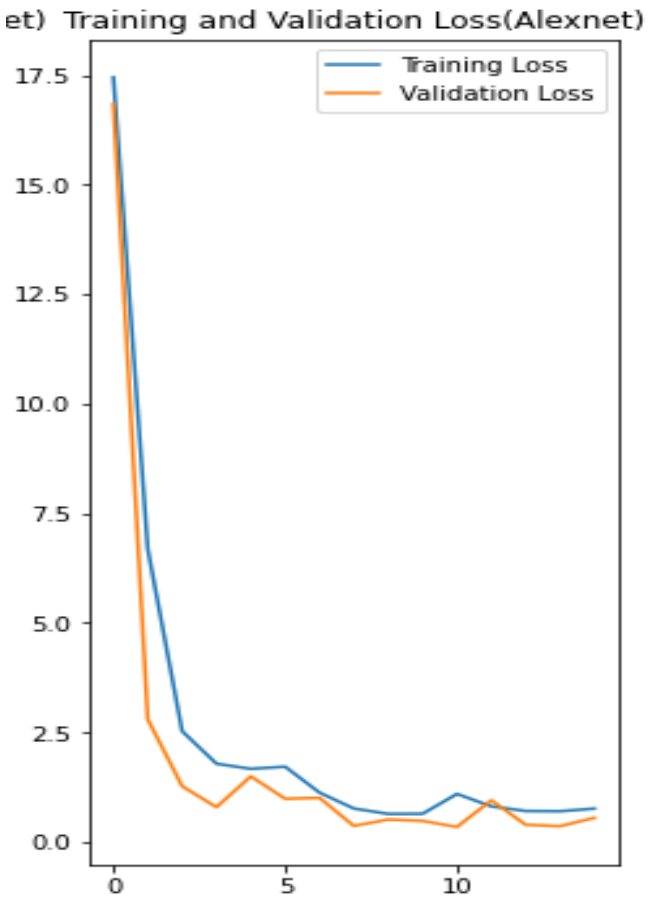

Fig6 Alex net plot for Training vs Validation Loss

The next model implemented for detection and classification of keratoconus was VGG 19,The accuracy for training vs validation plot is represented in fig 7 and Training vs validation loss plot is represented in fig 8.The model was trained and it yielded training accuray of $94.76 \%$ and validation acccuracy of $77.05 \%$, training loss and vaidation loss of 0.175 and 0.846 .

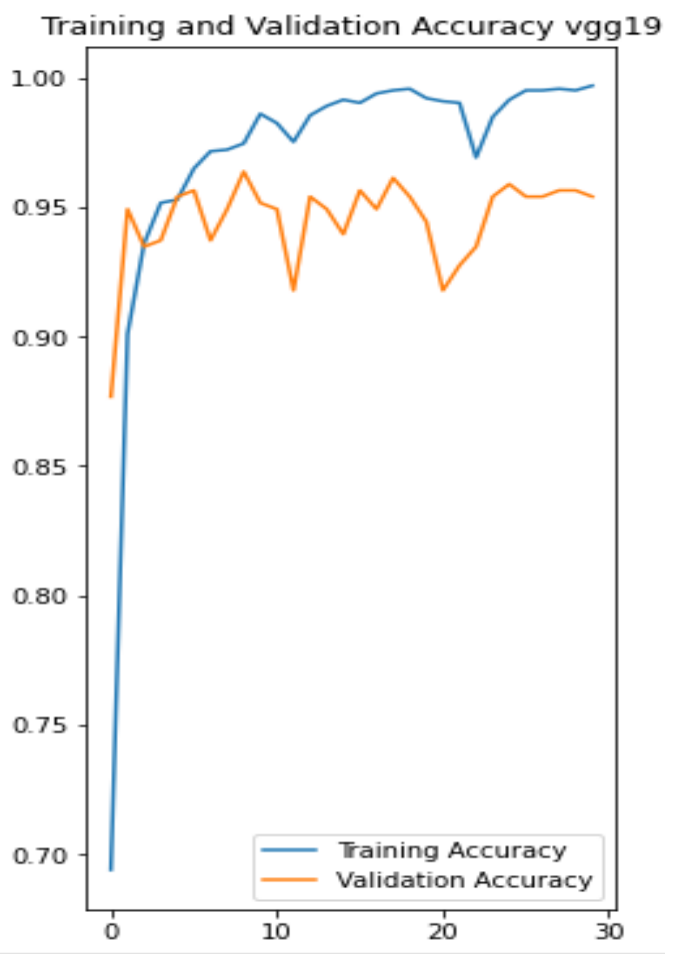

Fig 7 Training vs Validation Accuracy plot for Vgg 19 


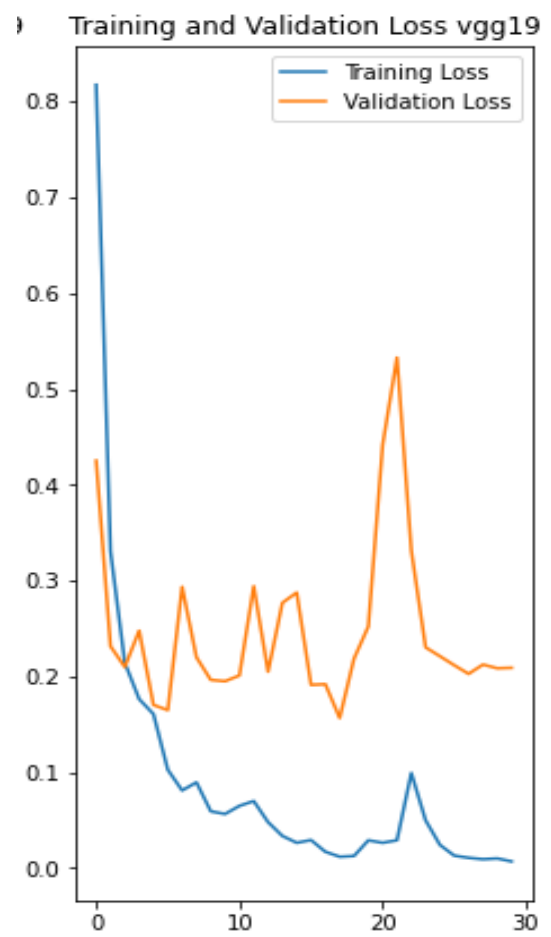

Fig 8Training Vs Validation loss plot for VGG 19

To summarize the results of classification model table 3 and 4 depicts the training and validation accuracies of each CNN models

Table 3 Model accuracies for training

\begin{tabular}{|l|l|}
\hline Model & Training Accuracy \\
\hline CON-KER CNN & $96.20 \%$ \\
\hline AlexNet & $88.90 \%$ \\
\hline Vgg 19 & $94.76 \%$ \\
\hline
\end{tabular}

Table 4 Model accuracies for validation

\begin{tabular}{|l|l|}
\hline Model & Validation Accuracy \\
\hline CON-KER model & $94.60 \%$ \\
\hline AlexNet & $84.78 \%$ \\
\hline Vgg 19 & $77.05 \%$ \\
\hline
\end{tabular}




\section{CONCLUSION AND FUTURE SCOPE}

The main goal of the project was to detect the keratoconus and also classify it into normal eye,mild, moderate and advanced Keratoconus, from the inclusion of different algorithms for the work, the detection and classification was successfully carried out with an effective accuracy, the aim of detecting the mild keratoconus was successfully done, the work will help in early screening and diagnosis of the keratoconus which can lead to controlling the progression of the disorder. Convolution neural networks are effective tools in the screening of several disease in the medical field. The newly introduced CON-KER model gives an accuracy of $96.20 \%$ which is effective in the detection and classification process. The newly built and introduced model can be used as an add-on in the screening of the disorder.

Topographic maps were used to train the neural networks will help in detection of keratoconus proved to be effective. The algorithms implemented yielded an effective result which are promising.

The work can be further improved with increasing the number of data used for training, medical images hard to procure, the future enhancement relies on the inclusion of more topographic scans and implementing convolution neural network models. This work will act as add-on or extra utility for the ophthalmologists in detection and classification of keratoconus for proper treatment. Future work can be concentrated on developing a complete solution which will be a standalone for the detection of the Keratoconusand help in giving proper treatment.

\section{References}

[1] Keratoconus severity identification using unsupervised machine learning, Siamak YousefiID1,2*, Ebrahim Yousefi1, Hidenori TakahashiID3, Takahiko HayashiID4, Hironobu Tampo3, Satoru Inoda3, Yusuke Arai3, Penny Asbell1- 2018.

[2]. Keratoconus detection using deep learning of colour-coded maps with anterior segment optical coherence tomography: a diagnostic accuracy study by Kazutaka Kamiya,1 Yuji Ayatsuka,2 Yudai Kato,2 Fusako Fujimura,1 Masahide Takahashi,3 Nobuyuki Shoji,3 Yosai Mori,4 Kazunori Miyata4, 2019 Sep 27;9(9): e031313. doi: 10.1136/bmjopen-2019-031313.

[3]. Detection of Keratoconus and Suspect Keratoconus by Machine Vision Fatemeh by Toutounchian, Jamshid Shanbehzadeh, Mehdi Khanlari.

[4]. Jack T. Holladay, MD, MSEE, FACS? Keratoconus Detection Using Corneal Topography, Journal of Refractive Surgery Volume 25 October (Suppl) 2009.

[5]. Lavric, Alexandru \& Valentin, Popa. (2019). KeratoDetect: Keratoconus Detection Algorithm Using Convolutional Neural Networks. Computational Intelligence and Neuroscience. $2019 . \quad 1-9$. $10.1155 / 2019 / 8162567$.

[6]. Early Tomographic Changes in the Eyes of Patients with Keratoconus by Mehdi Shajari, MD; Irfan Jaffary, MD; Kim Herrmann; Claudia Grunwald, MD; Gernot Steinwender, MD; Wolfgang J. Mayer, MD, PhD; Thomas Kohnen, MD, PhD, FEBO

[7]. Antonio Martínez-Abad,David P.Piñero New perspectives on the detection and progression of keratoconus, Journal of Cataract \& Refractive Surgery,Volume 43, Issue 9, September 2017, Pages 1213- 1227

[8]. Cavas-Martínez F, Bataille L, Fernández-Pacheco DG, Cañavate FJF, Alió JL. A new approach to keratoconus detection based on corneal morphogeometric analysis. PLoS One. 2017;12(9):e0184569. Published 2017 Sep 8. doi: 10.1371/journal.pone.0184569

[9] D. Anderson, “Understanding corneal topography," Paraoptometric Resource Center, vol. 1, pp. 1-12, 2000 [10]International Journal of Advanced Science and TechnologyVol.132 (2019), pp.1118http://dx.doi.org/10.33832/ijast.2019.132.02ISSN: 2207-6360IJAS00T Copyright $\quad$ C2019

\section{NADIAAUTOMATED IDENTIFICATION OF DIABETIC RETINOPATHY USING ALEXNET}

\section{ARCHITECTURE}

[11] R. Feng et al., "KerNet: A Novel Deep Learning Approach for Keratoconus and Sub-clinical Keratoconus Detection Based on Raw Data of the Pentacam System," in IEEE Journal of Biomedical and Health Informatics, doi: 10.1109/JBHI.2021.3079430. 
[12]. M. M. Daud, W. M. D. W. Zaki, A. Hussain and H. A. Mutalib, "Keratoconus Detection Using the Fusion Features of Anterior and Lateral Segment Photographed Images," in IEEE Access, vol. 8, pp. 142282-142294, 2020, doi: 10.1109/ACCESS.2020.3012583.

[13].A. Lavric, V. Popa, C. David and C. C. Paval, "Keratoconus Detection Algorithm using Convolutional Neural Networks: Challenges," 2019 11th International Conference on Electronics, Computers and Artificial Intelligence (ECAI), 2019, pp. 1-4, doi: 10.1109/ECAI46879.2019.9042100.

[14]. Espandar L, Meyer J. Keratoconus: overview and update on treatment. Middle East Afr J Ophthalmol. 2010;17(1):15-20. doi:10.4103/0974-9233.61212

[15] Mehrdad Mohammadpour, Zahra Heidari, Hassan Hashemi,Updates on Managements for Keratoconus,Journal of Current Ophthalmology,Volume 30, Issue 2,2018,Pages 110-124,ISSN 2452-2325

[16] M.A. Valdés-Mas, J.D. Martín-Guerrero, M.J. Rupérez, F. Pastor, C. Dualde, C. Monserrat, C. PerisMartínez,A new approach based on Machine Learning for predicting corneal curvature (K1) and astigmatism in patients with keratoconus after intracorneal ring implantation,Computer Methods and Programs in Biomedicine, Volume 116, Issue 1,2014,ISSN 0169-2607,

[17]. Ruiz Hidalgo I, Rozema JJ, Saad A, et al. Validation of an objective keratoconus detection system implemented in a scheimpflug Tomographer and comparison with other methods. Cornea2017;36:689-95.

[18] Issarti I, Consejo A, Jiménez-García M, et al. Computer aided diagnosis for suspect keratoconus detection. Comput Biol Med 2019; 109:33-42

[19]. E. Pinos-Vélez, M. Baculima-Pintado, M. Cruz-Cabrera and L. Serpa-Andrade, "Modeling of the human eye as a tool to determine the degree of involvement of keratoconus using the image processing," 2017 IEEE International Systems Engineering Symposium (ISSE), 2017, pp. 1-5, doi: 10.1109/SysEng.2017.8088267.

[20].A. Lavric et al., "Keratoconus severity detection from elevation, topography and pachymetry raw data using a machine learning approach," in IEEE Access, doi: 10.1109/ACCESS.2021.3086021.

[21]. Arbelaez MC, Versaci F, Vestri G, Barboni P, Savini G. Use of a support vector machine for keratoconus and subclinical keratoconus detection by topographic and tomographic data. Ophthalmology. 2012 Nov;119(11):2231-8. doi: 10.1016/j.ophtha.2012.06.005. Epub 2012 Aug 11. PMID: 22892148.

[22] N. Hallett et al., "Deep Learning Based Unsupervised and Semi-supervised Classification for Keratoconus," 2020 International Joint Conference on Neural Networks (IJCNN), 2020, pp. 1-7, doi: 10.1109/IJCNN48605.2020.9206694.

[23] N. Tang, H. Liu, K. Yue, W. Li and X. Yue, "Automatic classification for corneal ulcer using a modified VGG network," 2020 International Conference on Artificial Intelligence and Computer Engineering (ICAICE), 2020, pp. 120-123, doi: 10.1109/ICAICE51518.2020.00029.

[24] F. Nasrin, R. V. Iyer and S. M. Mathews, "Simultaneous Estimation of Corneal Topography, Pachymetry, and Curvature," in IEEE Transactions on Medical Imaging, vol. 37, no. 11, pp. 2463-2473, Nov. 2018, doi: 10.1109/TMI.2018.2836304.

[25] D. K. Elswah, A. A. Elnakib and H. El-din Moustafa, "Automated Diabetic Retinopathy Grading using Resnet," 2020 37th National Radio Science Conference (NRSC), 2020, pp. 248-254, doi: 10.1109/NRSC49500.2020.9235098.

[26] S. A. Hasan and M. Singh, "Automatic diagnosis of astigmatism for Pentacam sagittal maps," 2014 International Conference on Advances in Computing, Communications and Informatics (ICACCI), 2014, pp. 472-478, doi: 10.1109/ICACCI.2014.6968539.

[27] V. Galvis, T. Sherwin, A. Tello, J. Merayo, R. Barrera, and A. Acera, “'Ker-atoconus: An inflammatory disorder?' 'Eye, vol. 29, no. 7, pp. 843-859,Jul. 2015.

[28] . Georgiou, C. L. Funnell, A. Cassels-Brown, and R. O’Conor, “'Influence of ethnic origin on the incidence of keratoconus and associated atopic disease in Asians and white patients,'”Eye, vol. 18, no. 4, pp. 379383,Apr. 2004.

[29] . O. Kok, G. F. L. Tan, and S. C. Loon, “'Review: Keratoconus in Asia,’'Cornea, vol. 31, no. 5, pp. 581593, 2012.

[30] Tsubota K, Mashima Y, Murata H, Sato N, Ogata T. Corneal epithelium in keratoconus. Cornea 1995;14:77-83.

[31] .Chang HY, Chodosh J. The genetics of keratoconus.Semin Ophthalmol.2013;28(5-6):275e280.

[32] Hallermann W, Wilson EJ. Genetic aspects of keratoconus (author'stransl).Klin Monbl Augenheilkd. 1977;170(6):906e908.

[33] .Shneor E, Millodot M, Blumberg S, Ortenberg I, Behrman S, Gordon-Shaag A. Characteristics of 244 patients with keratoconus seen in anoptometric contact lens practice.Clin Exp Optom. 2013;96(2):219e224.

[34]M.Twa,J.Nichols,C.Joslin,P.Kollbaum,T.Edrington,M.Bullimore,G.Mitchell,K.Cruickshanks,D.Schanzlin,C haracteristicsofcornealectasiaafterlasikformyopia,Cornea23(5)(2004)447-457. 
[35]J.B.Randleman,M.Woodward,M.J.Lynn,R.D.Stulting,Riskassessmentforectasiaaftercornealrefractivesurgery ,Ophthalmology115(1)(2008)37-50.

[36] Wollensak G, Spoerl E, Seiler T. Riboflavin/ultraviolet-A-inducedcollagen crosslinking for the treatment of keratoconus.Am J Ophthalmol.2003;135:620-627

[37] R. C. Date, S. J. Jesudasen, C. Y. Wenget al., "Applications of deeplearning and artificial intelligence in retina,’International OphthalmologyClinics, vol. 59, no. 1, pp. 39-57, 2019.

[38] T. Seiler and A. Quurke, "Iatrogenic keratectasia after lasik ina case of forme fruste keraoconus,"J Cataract Refract Surg,vol. 24, pp. 1007-1009, Jul. 1998

[39] Rabinowitz, "Diagnosing keratoconus and patients at risk,"Cataract and Refractive Surgery Today, pp. 8587, May 2007.

[40] C. J. Rapuano, “Prevention of Iatrogenic Keratectasia,”KlinMonbl Augenheilkd, vol. 233, no. 6, pp. 695700, Jun 2016

[41] I. Issarti, A. Consejo, M. Jim' enez-Garć 1a, S. Hershko, C. Koppen,and J. J. Rozema, "Computer aided diagnosis for suspect keratoconusdetection,”Computers in Biology and Medicine, vol. 109, pp. 33-42,2019. 